\title{
HIGHLIGHTS
}

MOTILITY

\section{Colon motor function varies in chronic constipation}

US researchers have characterized different chronic constipation phenotypes by using a barostat manometric assembly inserted into the colon. "Chronic constipation is a common problem and the relationship between colonic transit and motor functions is poorly understood," explains Adil Bharucha from the Mayo Clinic in Rochester, Minnesota. "Our study showed that $53 \%$ of patients with slow transit constipation had normal fasting and postprandial colonic motor activity while $40 \%$ of patients with normal transit constipation had reduced fasting and/or postprandial colonic motor activity," he explains.

Patients with chronic constipation are classified into three groups according to their colonic transit and anorectal functions: normal transit constipation (NTC), isolated slow transit constipation (STC) and functional defecatory disorders (DD) in which transit in the colon is either normal or slow. This classification assumes that slow transit in the colon reflects problems with motor function. Previous studies on colonic motor activity and transit in chronic constipation have been limited to patients with STC and did not classify whether patients had DD. In addition, these studies evaluated phasic pressure activity, but not colonic tone or pressure-volume relationships (compliance) in chronic constipation.

The investigators assessed colon motor functions in 35 healthy women and 111 women who had NTC, STC or DD. Manometric sensors measured phasic pressure activity and a barostat balloon measured tone and compliance of participants' colons under fasting conditions, after a meal and after a colonic stimulant.

The researchers found that fasting and postprandial colon motor activity was reduced in just under half of patients with chronic constipation, irrespective of their classification, compared with healthy people. In addition, colon compliance was significantly reduced in patients with STC and DD but not NTC. The researchers demonstrated that patients could be subtyped into one of four groups, which were characterized by fasting and postprandial phasic activity and tone, postprandial high amplitude contractions and postprandial tonic responses in the colon.

"These assessments ... reveal four distinct phenotypes, defined by colonic motor abnormalities, in patients with chronic constipation. Colonic transit is an imperfect surrogate marker for colonic motor activity in chronic constipation,"

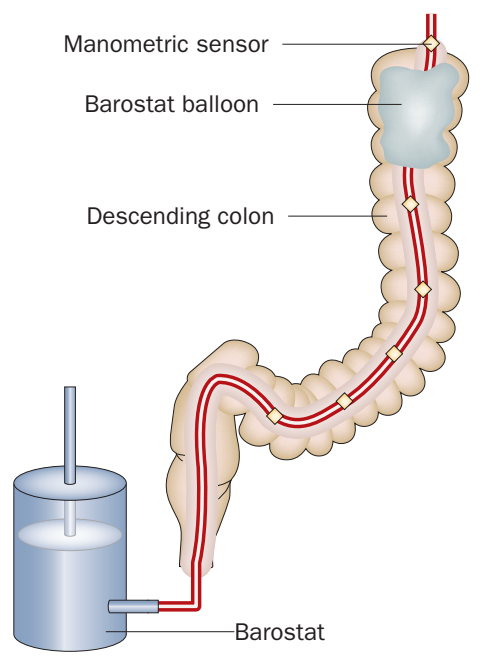

A barostat manometric assembly inserted into the colon.

says Bharucha, who suggests that future studies should assess colon motor functions after medical or surgical therapy and determine whether the different chronic constipation phenotypes can predict neuropathological abnormalities in the colon.

\section{Katrina Ray}

Original article Ravi, K. et al. Phenotypic variation of colonic motor functions in chronic constipation. Gastroenterology 138, 89-97 (2010) 